\title{
Exact Solutions for Time-Dependent Schrödinger Equation in Presence of the Pöschl-Teller Double-Ring Shaped Harmonic Potential
}

\author{
H. SOBHANi* AND H. HASSANABADi \\ Faculty of Physics, Shahrood University of Technology, P.O. Box 3619995161-316, Shahrood, Iran \\ (Received August 28, 2016; revised version April 8, 2019; in final form April 14, 2019) \\ In this paper, time-dependent Schrödinger equation is considered with the Pöschl-Teller double-ring shaped \\ harmonic potential. Then, for investigation of such a system, the Lewis-Riesenfeld dynamical invariant method is \\ presented and shown that how it works. After that, the form and eigenfunctions for the Lewis-Riesenfeld dynamical \\ invariant are derived and the exact solutions are studied. Then the physical interpretations of derived results are
} discussed.

DOI: 10.12693/APhysPolA.136.17

PACS/topics: time-dependent Schrödinger equation, Pöschl-Teller double-ring shaped harmonic potential, LewisRiesenfeld dynamical invariant

\section{Introduction}

Confining physical problems to systems which have interactions that only depend on one kind of variable takes us far from what is happening in nature. It is obvious that if we want to write a model of a system in the physics, we should regard everything that can affect the system, but analysis of such a system has its own difficulties, the main of them being absences of a smooth mathematical approach to investigate them. On the other hand, if we combine different methods to study such a system, we can find a handle on them. Let us illustrate our goal. We can consider that interactions consist of two identical parts: the coordinate-dependent and time-dependent part. If these parts can be separated, then we can deal with a problem that is less difficult than the non-separable case. In such situations, regarding specific interactions, the system can be analyzed easier than before. For example, we have collected some articles in which physical systems have been investigated that have radial-dependent interactions. In these papers, radial interactions have been studied in various conditions. It is fruitful to indicate that among those problems are: investigation of spin-less Salpeter equation with generalized Hulthen potential [1], the relativistic scattering states of the Hellmann potential [2], the Dirac equation under scalar and vector generalized isotonic oscillators and the Cornell tensor interaction [3], a new model for calculating the binding energy of the lithium nucleus under the generalized Yukawa and Hellmann potential [4], the Dirac oscillator problem in the presence of the Aharonov-Bohm effect with the harmonic potential in commutative and non-commutative

*corresponding author; e-mail: hadisobhani8637@gmail.com spaces [5], and relativistic symmetries of a multiparameter exponential-type potential within Coulomb-like and Yukawa-like tensor interactions [6]. For polar and azimuthal interactions also there have been a lot of efforts. These problems also have been studied in different situations. It is instructive that we mention here. These are, spin-orbit coupling for motion of a particle in a ring-shaped potential [7], a ring-shaped potential and its dynamical invariance algebra [8], an algebraic approach to the ring-shaped non-spherical oscillator [9], quantum properties of complete solutions for a new non-central ring-shaped potential [10], exact solutions of the Klein-Gordon equation with scalar and vector ringshaped potentials [11], and others that can be found in Refs. [12-33].

Solving time-dependent problems directly in most cases are not possible because the differential equations which can describe the system are partial differential equation. Such equations can be solved in very special forms that are not suitable for our goal. Although, investigations of them can be handled indirectly in which algebra and quantum tools have been used. It is interesting to mention here some examples such as, canonical transformations and exact invariants for timedependent Hamiltonian systems [34], exact solutions for the time-dependent Hamiltonian in high-dimension and many-body problem [35], exact wave functions of time-dependent Hamiltonian systems involving quadratic and inverse quadratic terms [36], two-dimensional timedependent Hamiltonian systems with an exact invariant [37], dynamical algebraic approach and invariants for time-dependent Hamiltonian systems in two dimensions [38], and other interesting topics that are available in Refs. [39-44].

In this article, we want to combine the conditions mentioned above to investigate a system which has timedependent 3-dimensional interaction in the spherical coordinates. So in what follows: in Sect. 2 the desired 
system has been introduced in detail. Then in Sect. 3 time-evolution for the system has been calculated and in Sect. 4, physical interpretations of derived results are presented.

\section{Schrödinger equation}

\section{with time-dependent 3-dimensional interaction}

The Schrödinger equation is the well-known equation which one finds in quantum mechanics. Output of this equation is called the wave function which has all information of the system that is under investigation. This equation has the form

$$
\mathrm{i} \hbar \frac{\partial \Psi(\boldsymbol{r}, t)}{\partial t}=H(\boldsymbol{r}, t) \Psi(\boldsymbol{r}, t)
$$

in which $H$ stands for Hamiltonian of system that can be written as

$$
H(\boldsymbol{r}, t)=\frac{P^{2}}{2 M(t)}+V(\boldsymbol{r}, t) .
$$

For our purpose we choose spherical coordinates because the interaction which we want to study is

$$
\frac{1}{2} M(t) \omega^{2}(t) r^{2}+\frac{U(\theta, \varphi)}{M(t) r^{2}}
$$

where

$$
\begin{aligned}
& U(\theta, \varphi)=V(\theta)+\frac{\alpha^{2} V(\varphi)}{\sin ^{2}(\theta)}, \\
& V(\theta)=\frac{b}{\sin ^{2}(\theta)}+\frac{A(A-1)}{\cos ^{2}(\theta)}, \\
& V(\varphi)=\frac{D(D-1)}{\sin ^{2}(\alpha \varphi)}+\frac{C(C-1)}{\cos ^{2}(\alpha \varphi)} .
\end{aligned}
$$

The $U(\theta, \varphi)$ is called the Pöschl-Teller double-ring shaped harmonic potential. So the total form of our desire system is

$$
\begin{gathered}
H(\boldsymbol{r}, t)=\frac{P^{2}}{2 M(t)}+\frac{1}{2} M(t) \omega^{2}(t) r^{2} \\
+\frac{1}{M(t) r^{2}}\left[\left(\frac{b}{\sin ^{2}(\theta)}+\frac{A(A-1)}{\cos ^{2}(\theta)}\right)\right. \\
\left.+\frac{\alpha^{2}}{\sin ^{2}(\theta)}\left(\frac{D(D-1)}{\sin ^{2}(\alpha \varphi)}+\frac{C(C-1)}{\cos ^{2}(\alpha \varphi)}\right)\right] .
\end{gathered}
$$

Now everything is ready to investigate time evolution of this system.

\section{Investigation of time evolution via Lewis-Riesenfeld dynamical invariant}

Before using this theory we should present a brief note about it. This theory enables us to investigate systems which have a time-dependent Hamiltonian. This method was initiated by Lewis and Riesenfeld [45]. They considered a time-dependent Hamiltonian and a dynamical invariant which satisfies

$$
\frac{\mathrm{d} I(t)}{\mathrm{d} t}=\frac{\partial I(t)}{\partial t}+\frac{1}{\mathrm{i} \hbar}[I(t), H(t)]=0,
$$

where $I(t)$ is a Hermitian operator. Operating with the left-hand of Eq. (3.1) on the $|\Psi\rangle$ and using Eq. (2.1), we can easily obtain

$$
\mathrm{i} \hbar \frac{\partial(I|\Psi\rangle)}{\partial t}=H(I|\Psi\rangle) .
$$

This implies that the action of the dynamical invariant on the ket produces other solutions of the Schrödinger equations. Therefore, because of being Hermitian, the eigenkets of $I(t)$ are orthogonal and complete set. It means that we can write $|\Psi\rangle$ in terms of eigenkets of $I(t)$. Mathematically, we can write

$$
I(t)|\lambda, \kappa\rangle=\lambda|\lambda, \kappa\rangle
$$

where $\lambda$ is eigenvalue of $I(t)$ which is time-independent, $\kappa$ stands for other quantum numbers, and $|\lambda, \kappa\rangle$ is the eigenket which is time-dependent. So for $I(t)$ we have

$$
|\Psi\rangle=\sum_{\lambda, \kappa} c_{\lambda, \kappa} \mathrm{e}^{\mathrm{i} \alpha_{\lambda, \kappa}(t)}|\lambda, \kappa ; t\rangle,
$$

with the constants $c_{\lambda, \kappa}$ and time-dependent phase factor $\alpha_{\lambda, \kappa}(t)$ that comes from

$$
\hbar \frac{\mathrm{d} \alpha_{\lambda, \kappa}(t)}{\mathrm{d} t}=\left\langle\lambda, \kappa\left|\mathrm{i} \hbar \frac{\partial}{\partial t}-H(t)\right| \lambda, \kappa\right\rangle .
$$

It should be noted that we are free to choose $\alpha_{\lambda, \kappa}(t)$ [45]. Now it is the time to turn back to our system, defining three operators as [46]:

$T_{1}=P^{2}+\frac{2 U(\theta, \varphi)}{r^{2}}, \quad T_{2}=r^{2}, \quad T_{3}=r P_{r}+P_{r} r$,

with $P_{r}=-\mathrm{i} \hbar\left(\frac{\partial}{\partial r}+\frac{1}{r}\right)$ and algebraic structures given below

$$
\begin{aligned}
& {\left[T_{1}, T_{2}\right]=-2 \mathrm{i} \hbar T_{3}, \quad\left[T_{2}, T_{3}\right]=4 \mathrm{i} \hbar T_{2},} \\
& {\left[T_{1}, T_{3}\right]=-4 \mathrm{i} \hbar T_{1} .}
\end{aligned}
$$

Following the method of Lewis-Riesenfeld [45, 46] we take the dynamical invariant as

$$
I(t)=\frac{1}{2}\left[\delta(t) T_{1}+\beta(t) T_{2}+\eta(t) T_{3}\right],
$$

in which $\delta(t), \beta(t)$ and $\eta(t)$ are arbitrary functions of time. Using Eqs. (3.6)-(3.8) and (3.1), we obtain

$$
\begin{aligned}
& I(t)=\frac{1}{2}\left[\left(\frac{1}{\rho^{2}}+M^{2} \dot{\rho}^{2}\right) r^{2}+\left(P^{2}+\frac{2 U(\theta, \varphi)}{r^{2}}\right) \rho^{2}\right. \\
& \left.\quad-\rho \dot{\rho} M\left(r P_{r}+P_{r} r\right)\right],
\end{aligned}
$$

where $\rho$ should satisfy

$$
\ddot{\rho}+\frac{\dot{M}}{M} \dot{\rho}+\omega^{2} \rho=\frac{1}{M^{2} \rho^{3}} .
$$

Introducing $\Phi(\boldsymbol{r}, t)=\langle\boldsymbol{r} \mid \lambda, \kappa\rangle$, Eq. (3.3) turns into

$$
I(t) \Phi(\boldsymbol{r}, t)=\lambda \Phi(\boldsymbol{r}, t) .
$$

To solve Eq. (3.11) it is better to use a unitary transformation in order to obtain simpler differential equation. The unitary transformation is

$$
\Phi(\boldsymbol{r}, t)=S \Phi^{\prime}(\boldsymbol{r}, t)=\exp \left(\frac{\mathrm{i} M \dot{\rho}}{2 \hbar \rho} r^{2}\right) \Phi^{\prime}(\boldsymbol{r}, t) .
$$

Consequently $I(t)$ turns into $I^{\prime}(t)=S^{\dagger} I(t) S$ which has form

$$
I^{\prime}(t)=\frac{1}{2}\left[\rho^{2}\left(P^{2}+\frac{2 U(\theta, \varphi)}{r^{2}}\right)+\frac{r^{2}}{\rho^{2}}\right],
$$

and therefore Eq. (3.11) changes to

$$
I^{\prime}(t) \Phi^{\prime}(\boldsymbol{r}, t)=\lambda \Phi^{\prime}(\boldsymbol{r}, t) .
$$


To simplify Eq. (3.14) we should define new variables as $X_{i}=\frac{x_{i}}{\rho}(i=1,2,3)$. Thus, by means of new variables Eq. (3.14) gives

$$
\begin{aligned}
& {\left[\frac{1}{R^{2}} \frac{\partial}{\partial R}\left(R^{2} \frac{\partial}{\partial R}\right)\right.} \\
& \left.\quad+\frac{1}{\hbar^{2}}\left(2 \lambda-\frac{L^{2}+2 U(\theta, \varphi)}{R^{2}}-R^{2}\right)\right] \Phi^{\prime}=0
\end{aligned}
$$

in which $R^{2}=\frac{r^{2}}{\rho^{2}}, \varphi=\tan ^{-1}\left(\frac{X_{2}}{X_{1}}\right)=\tan ^{-1}\left(\frac{x_{2}}{x_{1}}\right), \theta=$ $\cos ^{-1}\left(\frac{X_{3}}{R}\right)=\cos ^{-1}\left(\frac{x_{3}}{r}\right)$ and the $L^{2}$ operators are defined in spherical coordinate as:

$$
L^{2}=-\hbar^{2}\left(\frac{\partial^{2}}{\partial \theta^{2}}+\cot (\theta) \frac{\partial}{\partial \theta}+\frac{1}{\sin ^{2}(\theta)} \frac{\partial^{2}}{\partial \varphi^{2}}\right) .
$$

Using separation of variables method, Eq. (3.15) could be solved. So we set

$$
\Phi^{\prime}(R, \theta, \varphi)=\Gamma(R) H(\theta) K(\varphi),
$$

which causes Eq. (3.15) to break into the following differential equations:

$$
\begin{aligned}
& \frac{\mathrm{d}^{2} \Gamma(R)}{\mathrm{d} R^{2}}+\frac{2}{R} \frac{\mathrm{d} \Gamma(R)}{\mathrm{d} R}+\left(2 \lambda-R^{2}-\frac{\nu}{R^{2}}\right) \Gamma(R)=0, \\
& \frac{\mathrm{d}^{2} H(\theta)}{\mathrm{d} \theta^{2}}+\cot \theta \frac{\mathrm{d} H(\theta)}{\mathrm{d} \theta} \\
& +\left(\nu-\frac{\gamma+2 b}{\sin ^{2} \theta}-\frac{2 A(A-1)}{\cos ^{2} \theta}\right) H(\theta)=0 \\
& \frac{\mathrm{d}^{2} K(\varphi)}{\mathrm{d} \varphi^{2}}+\left(\gamma-\frac{2 \alpha^{2} D(D-1)}{\sin ^{2}(\alpha \varphi)}\right. \\
& \left.-\frac{2 \alpha^{2} C(C-1)}{\cos ^{2}(\alpha \varphi)}\right) K(\varphi)=0
\end{aligned}
$$

where we have used $\hbar=1$ for the sake of simplicity and separation constants are $\nu$ and $\gamma$. The differential equations above can be solved by Refs. [47, 48]. The solutions are

$$
\begin{aligned}
& \Gamma(R)=N_{R} \mathrm{e}^{-\frac{R^{2}}{2}}\left(R^{2}\right)^{\frac{1}{4}(\sqrt{8 \nu+1}-1)} L_{n}^{\frac{1}{2} \sqrt{8 \nu+1}}\left(R^{2}\right),(3.21) \\
& H(\theta)=N_{\theta}[\cos (\theta)]^{\frac{1}{2}(\sqrt{8(A-1) A+1}+1)}[\sin (\theta)]^{\sqrt{-4 A+2 b+\gamma}} \\
& \quad \times P_{n}^{\left(\frac{1}{2} \sqrt{8(A-1) A+1}, \sqrt{-4 A+2 b+\gamma}\right)}(-\cos (2 \theta)), \\
& K(\varphi)=N_{\varphi}[\cos (\alpha \phi)]^{\frac{1}{2}(\sqrt{1-8(C-1) C}+1)} \\
& \quad \times[\sin (\alpha \phi)]^{\frac{1}{2}(\sqrt{1-8(D-1) D}+1)} \\
& \quad \times P_{n}^{\left(\frac{1}{2} \sqrt{1-8(C-1) C}, \frac{1}{2} \sqrt{1-8(D-1) D}\right)}\left(1-2 \cos ^{2}(\alpha \phi)\right)
\end{aligned}
$$

where $N_{R}, N_{\theta}$ and $N_{\varphi}$ are normalization constants and

$$
\lambda=\frac{1}{2}\left(\sqrt{8 \nu+1}+4 n_{R}+2\right)
$$

$$
\begin{aligned}
\nu= & \sqrt{-(8(A-1) A+1)(4 A-2 b-\gamma)}+2 \sqrt{-4 A+2 b+\gamma} \\
& +2 n_{\theta}\left(2 \sqrt{-4 A+2 b+\gamma}+\sqrt{8(A-1) A+1}+2 n_{\theta}+2\right) \\
& +2(A-3) A+\sqrt{8(A-1) A+1}+2 b+\gamma+1, \quad(3.25) \\
\gamma= & -\frac{1}{2} \alpha^{2} \\
& \times\left[4 n_{\varphi}\left(\sqrt{1-8(c-1) c}+\sqrt{1-8(d-1) d}+2 n_{\varphi}+2\right)\right. \\
& +\sqrt{(8(c-1) c-1)(8(d-1) d-1)}-4(c-1) c \\
& +2 \sqrt{1-8(c-1) c}-4(d-1) d+2 \sqrt{1-8(d-1) d}+3] .
\end{aligned}
$$

Finally, for the phase factor we have

$$
\alpha(t)=-\frac{\lambda}{\hbar} \int_{0}^{t} \frac{\mathrm{d} t^{\prime}}{M\left(t^{\prime}\right) \rho^{2}\left(t^{\prime}\right)} .
$$

\section{Physical meaning of results}

If we want to understand treatments of the wave function we should find treatments of $\rho(t)$. Considering $\omega(t)=B(t)=\exp (-\lambda t)$, we have plotted this parameter using Eq. (3.10) in Fig. 1. In this figure parameters have been considered as $\lambda=2, \rho(0)=1$ and $\dot{\rho}(0)=0$. Recalling the situation of $\rho(t)$ and ascending essence of it, what is gained is that after passing time we lose the system. This is due to time-dependence of interactions. It means that as time passes the wave functions approaches to zero and it leads to destruction of the system.

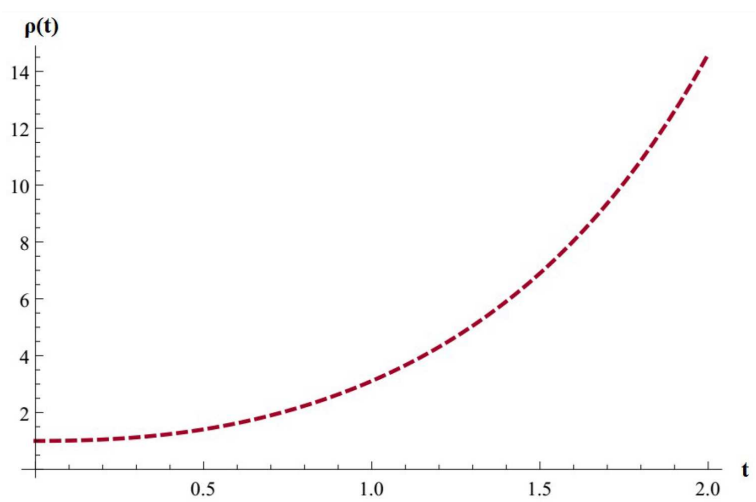

Fig. 1. Treatments of $\rho(t)$.

\section{Conclusion}

In this article, time evolution for a system with the Pöschl-Teller double-ring shaped potential was studied. This goal was achieved by using the Lewis-Riesenfeld 
dynamical invariant method. It enabled us to investigate time evolution for such a complicated system by establishing dynamical invariant and deriving its eigenfunctions and expanding wave function in terms of eigenfunctions of dynamical invariant. The details of the calculation to achieve the eigenfunctions were mentioned. Towards the end, physical interpretation of wave function was presented by plotting $\rho(t)$.

\section{References}

[1] A.N. Ikot, C.N. Isonguyo, Y.E. Chad-Umoren, H. Hassanabadi, Acta Phys. Pol. A 127, 674 (2015).

[2] B.H. Yazarloo, H. Mehrabana, H. Hassanabadi, Acta Phys. Pol. A 127, 684 (2015).

[3] H. Hassanabadi, E. Maghsoodi, A.N. Ikot, S. Zarrinkamar, Adv. High Energy Phys. 2014 831938 (2014).

[4] M. Ghazvini, N. Salehi, H. Hassanabadi, A.A. Rajabi, Chin. Phys. C 39, 063106 (2015).

[5] H. Hassanabadi, S.S. Hosseini, S. Zarrinkamar, Chin. Phys. C 38, 063104 (2014).

[6] H.P. Obong, A.N. Ikot, I.O. Owate, H. Hassanabadi, J. Kor. Phys. Soc. 66, 6 (2015).

[7] H. Hartmann, D. Schuch, Int. J. Quant. Chem. 18, 125 (2004).

[8] C. Quesne, J. Phys. A Math. Gen. 21, 3093 (1988).

[9] S.H. Dong, G.H. Sun, M.L. Cassou, Phys. Lett. A 328, 299 (2004).

[10] S.H. Dong, Ch. Y. Chen, M.L. Cassou1, Int. J. Quant. Chem. 105, 453 (2005).

[11] S.H. Dong, M.L. Cassou, Phys. Scr. 74, 285 (2006).

[12] X.A. Zhang, K. Chen, Z.L. Duan, Chin. Phys. 14, $42(2005)$.

[13] H.B. Filho, A.N. Vaidya, Phys. Lett. A 145, 69 (1990).

[14] F.L. Lu, C.Y. Chen, D.S. Sun, Chin. Phys. 14, 463 (2005).

[15] M.C. Zhang, G.Q. Huang-Fu, B. An, Phys. Scr. 80, 065018 (2009).

[16] M.C. Zhang, G.H. Sun, S.H. Dong, Phys. Lett. A 374, 704 (2010).

[17] M. Aktas, Int. J. Theor. Phys. 48, 2154 (2009).

[18] S.M. Ikhdair, R. Sever, Int. J. Theor. Phys. 46, 2384 (2007).

[19] A.D. Alhaidari, J. Phys. A Math. Gen. 38, 3409 (2005).

[20] F. Yasuk, C. Berkdemir, A. Berkdemir, J. Phys. A Math. Gen. 38, 6579 (2005).

[21] L. Chetouani, L. Guechi, T.F. Hammann, J. Math. Phys. 33, 3410 (1992).
[22] N. Barnea, W. Leidemann, G. Orlandini, Nucl. Phys. A 693, 565 (2001).

[23] H. Hartmann, Theor. Chim. Acta 24, 201 (1972).

[24] M.C. Zhang, Z.B. Wang, Chin. Phys. 16, 1863 (2007).

[25] G.F. Wei, S.H. Dong, Phys. Scr. 81, 035009 (2010).

[26] S.H. Dong, M. Lozada-Cassou, Phys. Scr. 74, 285 (2006).

[27] C.Y. Chen, Y. You, X.H. Wang, S.H. Dong, Phys. Lett. A 377, 1521 (2013).

[28] C.Y. Chen, F.L. Lu, D.S. Sun, S.H. Dong, Chin. Phys. B 22100302 (2013).

[29] D.S. Sun, Y. You, F.L. Lu, C.Y. Chen, S.H. Dong, Phys. Scr. 89045002 (2014).

[30] D.S. Sun, F.L. Lu, Y. You, C.Y. Chen, S.H. Dong, Mod. Phys. Lett. A 301550200 (2015).

[31] C.Y. Chen, F.L. Lu, D.S. Sun, Y. You, S.H. Dong, Ann. Phys. 371, 183 (2016).

[32] W. Li, C.Y. Chen, S.H. Dong, Adv. High Energy Phys. 2017, 7374256 (2017).

[33] Y. You, F.L. Lu, D.S. Sun, C.Y. Chen, S.H. Dong, Adv. High Energy Phys. 20188307486 (2018).

[34] J. Struckmeier, C. Riedel, Ann. Phys. 11, 15 (2002).

[35] Z.M. Bai, M.L. Ge, Phys. Lett. A 256, 159 (1999).

[36] J.R. Choi, Int. J. Theor. Phys. 42, 853 (2003).

[37] B. Grammaticos, B. Dorizzi, J. Math. Phys. 25, 2194 (1984).

[38] R.S. Kaushaland, S.C. Mishra, J. Math. Phys. 34, 5843 (1993).

[39] H. Sobhani, H. Hassanabadi, Eur. Phys. J. Plus 132 351 (2017).

[40] H. Sobhani, H. Hassanabadi, J. Kor. Phys. Soc. 71 , 8 (2017).

[41] H. Sobhani, H. Hassanabadi, J. Kor. Phys. Soc. 69 1509 (2017).

[42] H. Sobhani, H. Hassanabadi, Int. J. Mod. Phys. E 25, 1650073 (2016).

[43] L. Naderi, H. Hassanabadi, H. Sobhani, Int. J. Mod. Phys. E 25, 1650029 (2016).

[44] H. Sobhani, H. Hassanabadi, Phys. Lett. B 760, 1 (2016).

[45] H.R. Lewisand, W.B. Riesenfeld, J. Math. Phys. 10, 1458 (1969)

[46] M. Maamache, Ann. Phys. 254, 10 (1997).

[47] A.F. Nikiforov, V.B. Uvarov, Special Functions of Mathematical Physics, Birkhäuser, Basel 1988.

[48] A. Arda, R. Sever, C. Tezcan, Phys. Scr. 79, 015006 (2009). 